\title{
Enzymatic activity and immunoreactivity of Aca $s$ 4 , an alpha-amylase allergen from the storage mite Acarus siro
}

\author{
Jana Pytelková, Martin Lepšík, Miloslav Šanda, Pavel Talacko, Lucie Marešová and Michael Mareš*
}

\begin{abstract}
Background: Enzymatic allergens of storage mites that contaminate stored food products are poorly characterized. We describe biochemical and immunological properties of the native alpha-amylase allergen Aca s 4 from Acarus siro, a medically important storage mite.

Results: A. siro produced a high level of alpha-amylase activity attributed to Aca s 4 . This enzyme was purified and identified by protein sequencing and LC-MS/MS analysis. Aca $s 4$ showed a distinct inhibition pattern and an unusual alpha-amylolytic activity with low sensitivity to activation by chloride ions. Homology modeling of Aca s 4 revealed a structural change in the chloride-binding site that may account for this activation pattern. Aca s 4 was recognized by IgE from house dust mite-sensitive patients, and potential epitopes for cross-reactivity with house dust mite group 4 allergens were found.
\end{abstract}

Conclusions: We present the first protein-level characterization of a group 4 allergen from storage mites. Due to its high production and IgE reactivity, Aca s 4 is potentially relevant to allergic hypersensitivity.

Keywords: Aca s 4, Acarus siro, a-amylases, group 4 mite allergens, storage mites

\section{Background}

Storage mites are global pests of stored food products of increasing medical and economical impact. In agricultural environments, they cause occupational allergy in farmers and grain handlers. Storage mites are also found in house dust from rural and urban dwellings and are important contributors to the allergen content, which expands their clinical significance. The storage mites belong to the Acaridae and Glycyphagidae families; our work focuses on Acarus siro, one of the most frequent and abundant species in central Europe.

More than two dozen groups of mite-derived allergens have been described in the WHO/IUIS Allergen Nomenclature database http://www.allergen.org. Allergens from house dust mites of Dermatophagoides spp. have been extensively studied; however, much less is known about allergens from storage mites (e.g. 7 records for A. siro allergens) (for review, see [1-3]). There is

\footnotetext{
*Correspondence: mares@uochb.cas.cz

Institute of Organic Chemistry and Biochemistry, Academy of Sciences of the Czech Republic, 16610 Prague, Czech Republic
}

increasing evidence that mites contain epitopes that are species-specific as well as cross-reactive among species. The effect of a partial cross-reactivity between storage mites and house dust mites and co-sensitization by both groups further increases the medical impact of storage mites [4-9]. A detailed analysis of storage mite-derived allergens at the protein level will be necessary to better evaluate aspects of their sensitization specificity and biochemical activity, as well as to improve diagnosis and treatment.

Group 4 mite allergens are homologous proteins of the $\alpha$-amylase class $[10,11]$. Group 4 allergens have been investigated in house dust mites such as Dermatophagoides pteronyssinus, Euroglyphus maynei, and Blomia tropicalis, and their sequences have been determined [12-14]. The biochemical properties of Der p 4 were analyzed in detail, including its interaction with major cereal flour allergens that act as $\alpha$-amylase inhibitors $[14,15]$. The IgE-binding activity of group 4 allergens has been demonstrated for $\sim 30 \%$ of allergic subjects in Western populations and China [12-14];
C Biomed Central 
these allergens may also be the major contributor to the serum activity, as found in an Australian Aboriginal community [16]. In this work, we analyze native Aca s 4 from $A$. siro, the first $\alpha$-amylase allergen to be isolated from storage mites. Specifically, we describe its biochemical and immunological properties. Furthermore, we provide insight into the 3D structure of Aca s 4 with the help of a novel homology model, the first 3D model of a group 4 allergen.

\section{Results and Discussion}

\section{Quantification of $\alpha$-amylase activity in $A$. siro}

A high $\alpha$-amylase activity was demonstrated in the whole body extract from the storage mite $A$. siro using chromogenic starch as a substrate. Figure 1 shows that this activity was one order of magnitude higher than that measured for a model house dust mite $D$. farinae (specific activity $599.6 \pm 18.0$ and $64.1 \pm 0.3{\mathrm{U} . \mathrm{mg}^{-1} \text { pro- }}^{-}$ tein, respectively). In both species, a pronounced $\alpha$-amylase activity was also detected in the faecal extract, suggesting that $\alpha$-amylases are digestive enzymes secreted into the gut lumen and released in the mite faeces (Figure 1).

For comparison, the proteolytic activity was determined in the whole body extracts, which showed that $D$. farinae has a higher proteolytic activity than $A$. siro (specific activity $21.9 \pm 0.9$ and $16.5 \pm 0.4{\mathrm{U} . \mathrm{mg}^{-1} \text { pro- }}^{-}$ tein, respectively) and a higher content of cysteine

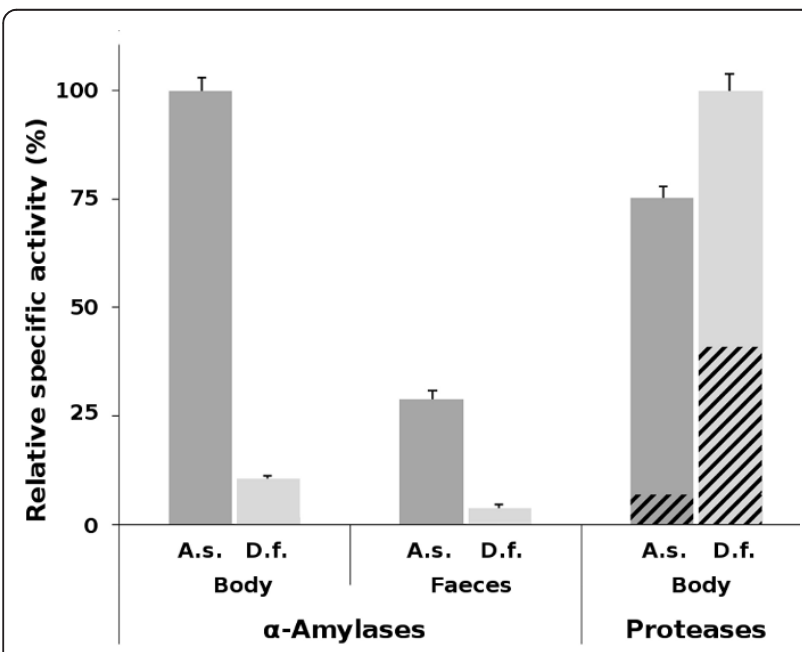

Figure 1 Distribution of $\alpha$-amylase and protease activities in the whole body extract and faecal extract of $A$. siro (A.s.) and D. farinae (D.f.). The $\alpha$-amylase activities were assayed at the respective $\mathrm{pH}$ optima with $\mathrm{RBB}$-starch as a substrate. The protease activities were assayed with azocasein as a substrate; the contribution of cysteine proteases (dashed) was determined as the part of protease activity inhibited by E-64. The specific activities (units per mg protein) are normalized to the maximum value measured for $\alpha$-amylases and proteases, respectively; mean values \pm SE are given. proteases (Figure 1). We conclude that there is an important difference in the distribution of digestive enzymes in these model species of storage and house dust mites, which most likely reflects their feeding ecology. The high level of $\alpha$-amylase activity in $A$. siro is in accordance with the feeding preferences of $A$. siro, a granivorous species evolutionarily adapted to utilization of a starch-rich diet [17].

\section{Isolation and proteomic identification of Aca s 4}

$\alpha$-Amylase was purified to homogeneity from the whole body extract of $A$. siro using an optimized procedure for affinity precipitation with glycogen. The typical yield was approximately $175 \mu \mathrm{g}$ from $1 \mathrm{~g}$ of fresh weight of mites. The purified enzymatically active $\alpha$-amylase migrated as a single band of $56 \mathrm{kDa}$ on SDS-PAGE (Figure 2). We performed a two-pronged proteomic characterization of this protein: (i) the $\mathrm{N}$-terminal amino acid sequence, XSPYSNPHFTGSR ( $\mathrm{X}$ is an unidentified residue), was determined by Edman sequencing and (ii) the protein was subjected to enzymatic digestion followed by LC-MS/MS analysis. The data were searched against the UniProt protein database, which revealed identity with the cDNA-derived protein sequence of an $A$. siro $\alpha$-amylase homolog denoted Aca s 4 (GenBank: ABL09312). The MS/MS peptide coverage of this sequence was $\sim 31 \%$ (Figure 3 ). A theoretical mass calculated for the mature Aca s 4 (sequence starting at the native $\mathrm{N}$-terminus) is $55956 \mathrm{Da}$, which is in good agreement with the experimental value obtained for the purified Aca s 4 (Figure 2).

\section{Biochemical functional characterization of Aca s 4}

The purified Aca s 4 was characterized with regard to its substrate and inhibitor interactions. The $\mathrm{pH}$ profile (Figure 4a) shows that the enzyme functions in the slightly acidic to neutral range, with a maximum at $\mathrm{pH}$ $\sim 6.5$, which is in accordance with the $\mathrm{pH}$ optimum of $\alpha$-amylase activity measured with the whole body extract of $A$. siro [17]. A similar $\mathrm{pH}$ optimum was also reported for the purified Der p 4 [14]. We tested the modulation of the Aca s 4 activity by chloride ions, which are general activators of animal $\alpha$-amylases $[18,19]$. Figure $4 \mathrm{~b}$ shows the activation of a typical chloride-dependent $\alpha$-amylase from porcine pancreas and of Aca s 4 in the presence of $\mathrm{NaCl}$. Chloride ions induced an increase in the activity of both Aca s 4 and its porcine homolog; however, Aca s 4 was activated to a lesser extent. Aca s 4 was inhibited by acarbose $\left(\mathrm{IC}_{50}\right.$ $\sim 3.8 \mu \mathrm{M}$ ), a microbial oligosaccharide that is a general inhibitor of enzymes of the $\alpha$-amylase class. This is in line with our previous finding that acarbose exerts an acaricidal activity against $A$. siro by inhibiting its digestive amylolytic activity [17]. Interestingly, Aca s 4 was 


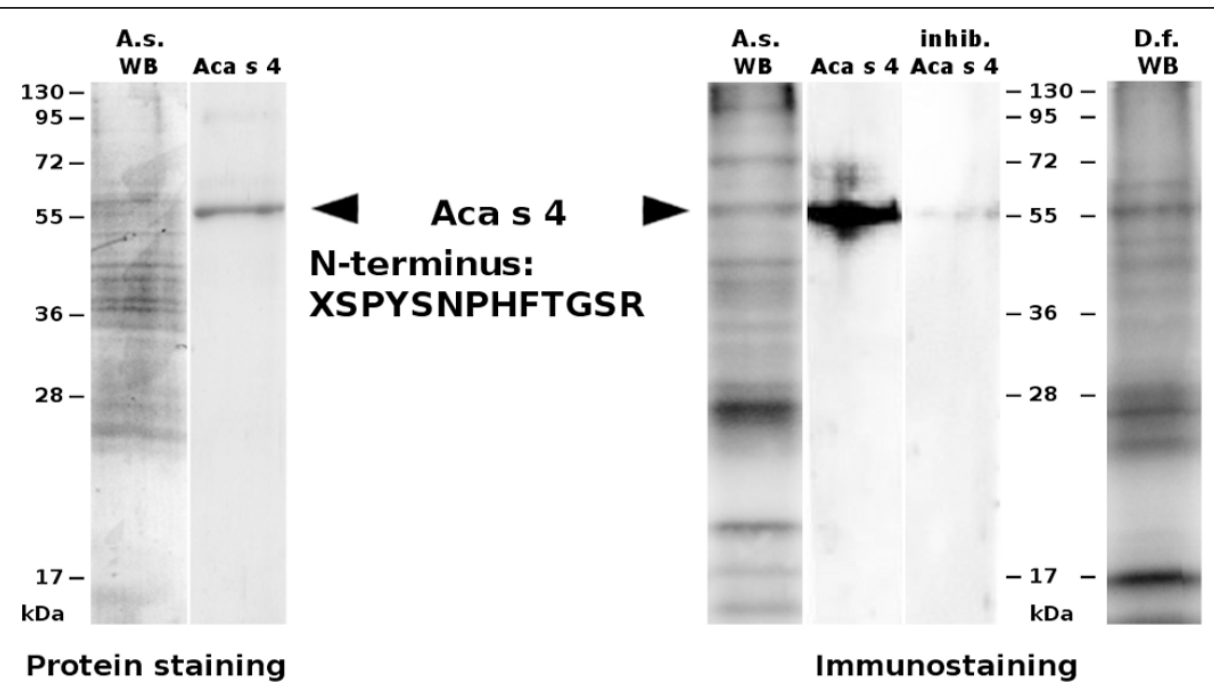

Figure 2 Purification and IgE reactivity of Aca s 4 . The whole body extracts (20 $\mu \mathrm{g})$ of A. siro (A.s WB) and D. farinae (D.f. WB) and the purified Aca s $4(2.5 \mu \mathrm{g})$ were resolved by SDS-PAGE. Left-hand panel: A gel stained for protein with Coomassie blue. Right-hand panel: Western blot probed with pooled sera from mite-allergic patients sensitive to Dermatophagoides spp. and with anti-lgE antibodies and developed by chemiluminescence. For immunostaining inhibition (inhib.), the pooled sera were preincubated with purified Aca $s 4$. The arrows mark the position of Aca s $4(\sim 56 \mathrm{kDa})$ with the $\mathrm{N}$-terminal sequence determined by Edman sequencing. Molecular mass standards are indicated.

insensitive to inhibition by two types of proteinaceous inhibitors of plant origin, namely wheat inhibitors WI-1 and WI-3 (tetrameric and monomeric form, respectively) and bean inhibitor $\alpha \mathrm{AI}-1$, which are potent inhibitors of various insect and mammalian $\alpha$-amylases $[20,21]$. A pronounced inhibitory effect against Der p 4 has been reported for the tetrameric wheat inhibitor [15]. We applied a combinatorial library of synthetic PAMIs (Peptide $\alpha$-Amylase Inhibitors) that was developed to analyze the inhibitory specificity of $\alpha$-amylases [22]. Using this tool, we compared the inhibitory specificity of Aca s 4 and Der $\mathrm{f} 4$ (an $\alpha$-amylase of the house dust mite $D$. farinae, measured in the extract). Figure 4c shows that inhibition profiles of both enzymes follow the same general trend but also have distinct features. This result indicates that the active site regions of mite $\alpha$-amylases share overall architecture but differ in some structural details. This analysis helps increase understanding of the different affinities of mite $\alpha$-amylases to natural proteinaceous inhibitors such as the wheat $\alpha$ amylase inhibitor [15].

\section{Three-dimensional model of Aca s 4}

A structural model of Aca s 4 was created by homology modeling (see Methods for details). The Aca s 4 structure shows an overall fold and secondary structure elements forming three consensus domains as in the insect and mammalian $\alpha$-amylases (Figure 5a) $[10,11]$. The disulfide pattern is composed of four conserved disulfides and one additional disulfide (Cys113-Cys126) located in the B domain (Figure 3); Aca s 4 does not contain free- thiol cysteines, as demonstrated experimentally by a thiol labeling experiment (see Methods, data not shown). The catalytic center of Aca s 4 consists of three acidic residues and retains the characteristic $\alpha$-amylase architecture (Figure 5a). We inspected the calcium-binding and chloride-binding sites, which are known to be important for active site function in the animal $\alpha$-amylases. The structure of the calcium-binding site, which is necessary for the stabilization of the active site, is preserved in the Aca s 4 model. In contrast, the chloridebinding site of Aca $s 4$ was found to be modified in comparison with the structures of other animal $\alpha$-amylases. The typical chloride-binding residues Arg194, Asn295 and Arg334 (Aca s 4 numbering) forming the "RNR signature" are not all conserved, and Asn295 is replaced by Ser in Aca s 4 (Figure 3). Furthermore, a comparison of the Aca s 4 sequence with those of its homologs available in the sequence database showed that this substitution (resulting in RSR signature of the chloride-binding site) is specific for $\alpha$-amylases of mite origin (Figure 3 ). The chloride ion serves as an allosteric activator of catalysis of $\alpha$-amylases $[18,19]$. The lower sensitivity of Aca s 4 to chloride activation (Figure 4a) is likely due to the substitution of Asn with Ser in the chloride-binding site. This is supported by a study performed on human pancreatic $\alpha$-amylase in which the chloride-binding residues were mutated [18]; the substitution at position 295 resulted in a defect in catalytic efficiency and chloride binding that resembles the behavior of Aca s 4. Recently, we have described another evolutionarily acquired mutation (Arg-to-Gln334) in the 


\section{Aca $s 4$ \\ Tyr p 4 \\ Blo $t 4$ \\ Der $p 4$ \\ Eur m 4 \\ HPA \\ Aca 54 \\ Tyr p 4 \\ Blo $t 4$ \\ Der $p 4$ \\ Eur m 4 \\ HPA}

Aca $s 4$

Tyr p 4

Blo t 4

Der $p 4$

Eur m 4

HPA

Aca $s 4$

Tyr p 4

Blo $t 4$

Der $p 4$

Eur m 4

HPA

Aca $s 4$

Tyr p 4

Blo $t 4$

Der $p 4$

Eur m 4

HPA

Aca $s 4$

Tyr p 4

Blo $t 4$

Der $p 4$

Eur m 4

HPA

Aca $s 4$

Tyr p 4

Blo $t 4$

Der $p 4$

Eur m 4

HPA

Aca 54

Tyr p 4

Blo $t 4$

Der $p 4$

Eur m 4

HPA

GSPYSNPHFTGSRSVITHLMQWKFDDIAAECERFLGPKGYGGIQLSPVNEHAVLGN--RP 58 NSP YSHP HF AGNRSVIVHLMQWKFVDIAAECERF LGP F GYGGIQLSPVNEHAVLGN--RP 58 -SPYSDPHFOHNRKVITHLMOWKFVDIADECERFLGPYGYGGVQVSPVNEHASLDR--HP 57 --KYHNP HF I GNRSVITHLMEWKYDD I GDECERFLGPYGYGGVQVSPVNEHAILDR--RP 56 --KYHNP HF I GNRCVI THLMEWKYDD I GDECERF LGPYGYGGVQVSPVNEHAILDR--RP 56 ---QYSPNTQQGRTSIVHLFEWRWVDIALECERYLAP KGFGGVQVSPPNENVAIYNPFRP 57

WYELYQPVGYKIQSRSGNEEQFKGMVQRCNKVGVRIYVDIVMNHMSGAQEGHGN---CWF 115 WYELYQPVSYRI ISRSGNEAEFKDMVHRCNKAGVRI YVDAVLNHMTGAQEGSG---TAGS 115 WYELYQPVSYRIVSRSGTESQFRDMVHRCNKAGVRIYVDVVLNHMTGPQSGVGI---DGT 114 WYERYOPVSYDIRTRSGDEOOFRRMVKRCNKAGVRIYVDIVLNHMTGAOSGKGT---NGH 113 WYERYQPVSYDLRTRSGDEQQFRRMVKRCNKAGVRIYVDIVLNHMTGGQSGQGT---NGN 113 WWERYQPVSYKLCTRSGNEDEFRNMVTRCNNVGVRIYVDAVINHMCGNAVSAGTSSTCGS 117

\section{$+\quad \neq \quad \neq \quad$ *}

KLQWHHDVSRCSLLVPNDFHGRESCHTDNMDIKNYDNPEEARNCRLSGLRDLKQSSEYVR 175 HYNGNSMQYPGVPF GPNDF HGHESCPTSDLEI HNYDNAQEARNCRLSGLRDLKQSSEYVR 175 HYDGNSMOYPGIPFGPNDFHGHESCPTSNLDIQNYDDPTQARNCRLSGLRDLKQSADYVR 174 HYDGNTLOYPGVPFGPNDFHGHESCPTODLEIHDYTNP KEARNCRLSGLRDLKOOSEYVR 173 HYNGI TMQYPGVPF GPNDFHGHESCQTKDLEI HNYSDRNEARNCRLSGLRDLKQQSEYVR 173 YFNPGSRDFPAVPYSGWDFNDGK-CKTGSGDIENYNDATQVRDCRLTGLLDLALEKDYVR 176

$$
\text { \# }
$$

e

QKOADFLNHLIDLGVAGSRSDASKHMWPGDLEAIYGKLHNLNTAYFPANSRPFIYHEVIY 235 TKQAEFLNKLIDAGVAGFRFDASKHMWPGDLEAIYGKLHSLNTAYFPSNSKPFVYHEVIY 235 TKQADFLNHLIDIGVAGFRFDASKHMWPGDLQAIYSKLHHLNEKYFP SNSNPF IYHEVIY 234 OKOVDF LNHLIDIGVAGF RSDASTHOWPDDLRSIYSRLHNLNKEFFPENSQPF IYHETIY 233 KKOVDFLNHLIDIGVAGFRSDASTHOWPDDLRSIYSOLHTLNKEFFPENSHPF IYHETIY 233 SKIAEYMNHLIDIGVAGFRLDASKHMWPGDIKAILDKLHNLNSNWFPAGSKPF IYQEVID 236

YGGDGIKSSDYTKLGRAIEFHFYRDIANVVRRHN--OLKTVKNFGOPWGMVPSDDALVMV 293 YGGNGIKSSEYTRLGRAIEFHYYRELANVLRHHN--QLKWLKTFGQNWSMVP SDDALVMV 293 SNNNAISISDYTKLGRS IEFHYYHELCNVIRGNN--KLKWLHNFGQPWGMVPNDDALIMV 292 YGGNGINSNEYTSLGRIIEFRFYKEITNVFRGNN--PLHWLKNFGTEWGLVP SGDALVMI 291 YGGNGINSNEYTSLGRI IEFRFYKEITNVFRGNN--OLRWLKNFGTEWGLVP SNDALVMI 291 LGGEP IKSSDYFGNGRVTEFKYGAKLGTVIRKWNGEKMSYLKNWGEGWGFVPSDRALVFV 296

$$
\text { \# Q }
$$

DSHDLORFHTGOVGVNINYFESRLLKVATAFMLAWPYGVPRVMSSYHWDOKIEDGKDKND 353 DSHDMORGHTGTLGLNINFFEPKLLKVATAFMLAWPYGVTRVMSSYHWNQKIONGHDVND 353 DSHDLQRGHTGQLGLNINYFESRLLKVATAFMLAWPYGIPRVMSSYRWNQKIVNGKDEND 352 DSHDLRVGHTGKLGFNINCFEGRLLKAATAFMLAWNYGVPRVMSSYFWNQI IKDGKDVND 351 DSHDLRVGHTGKLGFNINCFEARLLKASTAFMLAWNYGIPRVMSSYFWNOI IRDGKDVND 351 DNHDNQRGHGAGGASILTFWDARL YKMAVGFMLAHPYGFTRVMSSYRWPRQFQNGNDVND 356

WIGPPSDGSGNILSVTPOPDDTCNKEWICEHRWROIYNMVHFRNVAGNEAVSHWWDNGDY 413 WMGPP SDSHGNILSVKPNADLTCNKEWVCEHRWROI HNMVAFRNIAGSEPVTHWWDNGDY 413 WIGPPADGSGSILSVKPNSDLTCNQEWICEHRWKQI YNMVQF RNTAGDEPVKNWWDNGDY 412 WVGPP SDKNGNILSVHPNPDMTCNHEWICEHRWREI YNMVKF RMIAGQEPVHNWWDNGDY 411 WVGP PTDQHGNILSVHPNADLTCNHEWICEHRWRQI YNMVRFKMIAGQEPVRNWWDNGDY 411 WVGPPNN-NGVIKEVTINPDTTCGNDWVCEHRWRQIRNMVIFRNVVDGQPFTNWYDNGSN 415

$\begin{array}{llll}\text { Aca s } 4 & \text { GNADINIAKTAEDPFVAIHVEAKLH- } & 496 \\ \text { Tyr p } 4 & \text { GNAEITINSSSDDDFVAIHVHAKH-- } 495 \\ \text { Blo t } 4 & \text { GHAMISIGANADDHL---------- } 485 \\ \text { Der p } 4 & \text { GQADVYVGHDEFDAFVAYHIGARIVS } 496 \\ \text { Eur m } 4 & \text { GLADIYVGHDELDAFVAYHIGARIE- } 496 \\ \text { HPA } & & \text { GKAHFSISNSAEDPFIAIHAESKL-- } 496\end{array}$

Figure 3 Multiple sequence alignment of Aca s 4 with other $\alpha$-amylases of mite origin and human $\alpha$-amylase. Aca s 4: Acarus siro (GenBank: ABL09312); Tyr p 4: Tyrophagus putrescentiae (GenBank: ABM53754); Blo t 4: Blomia tropicalis (GenBank: AAQ24543) [12]; Eur m 4: Euroglyphus maynei (GenBank: AAD38943) [13]; Der p 4: Dermatophagoides pteronyssinus (GenBank: AAD38942) [13]; HPA: human pancreatic $\alpha$ amylase (GenBank: AAH07060). The sequence similarities to Aca s 4 are $74 \%, 70 \%, 64 \%, 66 \%$, and $50 \%$, respectively. Full-length sequences of mature proteins are aligned. Amino acids identical to those of Aca $s 4$ are shaded. In the Aca s 4 sequence, the $\mathrm{N}$-terminal sequence determined by Edman sequencing (dotted underline) and fragments determined by LC-MS/MS analysis (solid underline) are indicated. Positions of catalytic residues (@) and residues binding the $\mathrm{Cl}^{-}(\#)$ and the $\mathrm{Ca}^{2+}\left(^{*}\right)$ are marked. N-glycosylation signals are double underlined (Aca s 4 and Blo t 4 have no predicted $\mathrm{N}$-glycosylation sites). Note the Asn295Ser mutation located in the $\mathrm{Cl}^{-}$-binding site of mite $\alpha$-amylases (compared to HPA and other animal $\alpha$-amylases). Cys residues forming four conserved disulfide bridges ( $\ddagger)$ and one specific disulfide (+) in the Aca $s 4$ molecule are marked. 

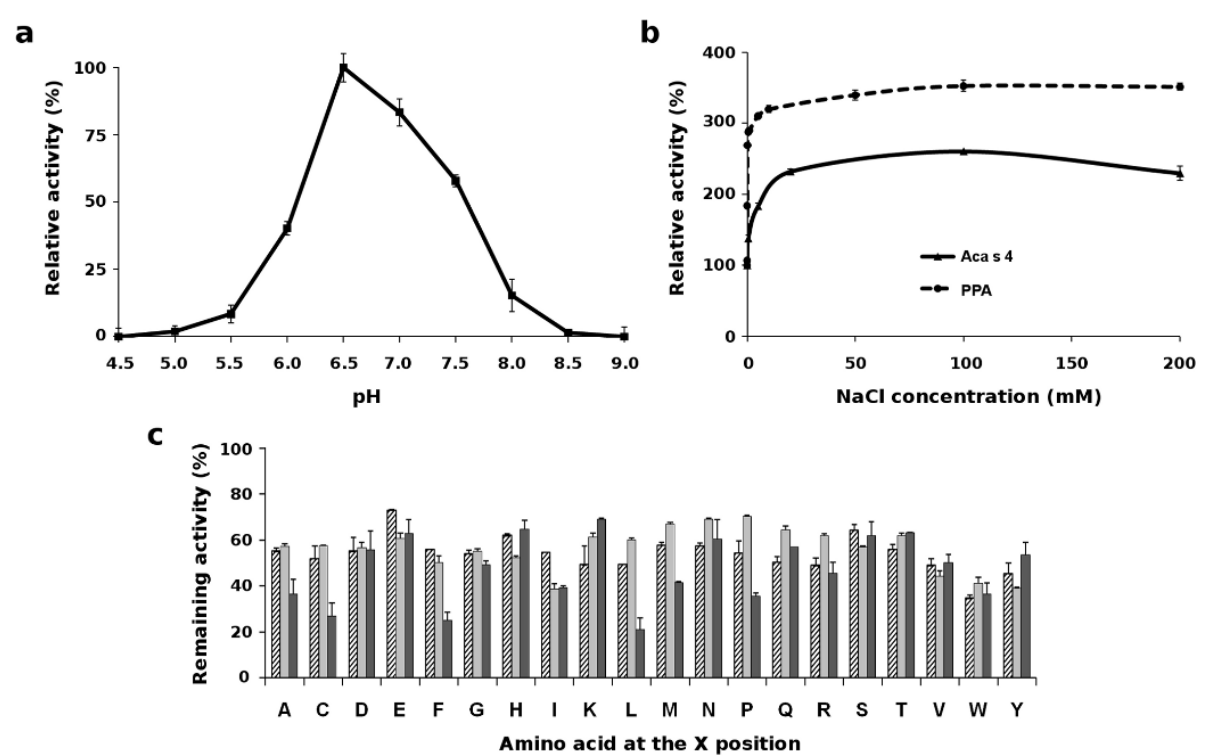

Figure 4 Enzymatic properties of purified Aca s 4. (a) pH profile determined with RBB-starch as a substrate. Mean values \pm SE are normalized to the maximum value. (b) Activation effect of $\mathrm{NaCl}$ on amylolytic activity of Aca s 4 (solid line) and porcine pancreatic $\alpha$-amylase (PPA) (dashed line) measured with the RBB-starch assay. Mean values \pm SE are expressed as a percentage of activity relative to the control without $\mathrm{NaCl}(100 \%)$. (c) Inhibition profile of Aca $s 4$ determined by a library of PAMls with the general structure Ac-XHWYYRCW-NH${ }_{2}$; the $\mathrm{X}$ position contains one of the 20 naturally occurring amino acids as indicated. The inhibition sensitivity of the purified Aca s 4 (hatched) is compared to that of whole body extracts of $A$. siro (gray) and D. farinae (black). Mean values \pm SE are expressed as the percentage of amylolytic activity (RBB-starch assay) relative to the uninhibited control $(100 \%)$.

chloride-binding site that leads to the chloride-independence of alkaline $\alpha$-amylases of lepidopteran insects [21].

\section{Immunoreactivity of Aca s 4}

The IgE reactivity of Aca s 4 was tested by immunoblotting using pooled sera from patients allergic to house dust mites (see Methods). Figure 2 shows binding of IgE to the purified Aca s 4; the staining was specific, as it was inhibited when using pooled sera preincubated with Aca $\mathrm{s} 4$. The staining pattern of the whole body extract of $A$. siro demonstrated that Aca s 4 of $\sim 56 \mathrm{kDa}$ is a significant IgE-reactive component although it is a protein of low abundance in the $A$. siro extract. Aca s 4 is likely recognized by serum IgE due to (i) a cross-reactivity with homologous $\alpha$-amylase allergens from house dust mites (see a $\sim 56 \mathrm{kDa}$ band immunostained in the whole body extract of $D$. farinae in Figure 2) and/or (ii) a reactivity of anti-Aca s 4 IgE induced by exposure to A. siro (most likely during co-sensitization with house dust mites). To gain insight into the structural basis of a possible cross-reactivity, we compared Aca s 4 with Der $\mathrm{p}$ 4 , an $\alpha$-amylase allergen from $D$. pteronyssinus with known sequence (GenBank: AAD38942; [13]). Both sequences display a $66 \%$ amino acid identity. The conserved sequence regions are shown on the surface model of Aca s 4 (Figure 5b); they form a dense net of clusters representing the potential common IgE-binding epitopes.

\section{Conclusions}

Our work provides the first comprehensive protein-level analysis of the $\alpha$-amylase allergen Aca s 4 from A. siro. The results give new insights into the biochemistry of the group 4 allergens of mites and suggest that the interaction of Aca s 4 with patients' IgE may be relevant to allergic hypersensitivity to mites.

\section{Methods \\ Materials}

Enzyme inhibitors: Acarbose was obtained from Bayer (Berlin, Germany); WI-1, WI-3 and E-64 from Sigma (St. Louis, MO); and Pefabloc from Roche (Indianapolis, IN). $\alpha$ AI-1 was isolated according to [20]; the development and synthesis of PAMIs is described in [22]. The substrates and enzymes: Remazol Brilliant Blue dyed starch (RBB-Starch) and azocasein were purchased from Fluka (Buchs, Switzerland), porcine pancreatic $\alpha$-amylase (PPA) from Sigma. The A. siro and D. farinae originated from laboratory cultures that were maintained and mass-reared as previously described [17,23]. Live mites were collected from the stock culture and washed; the faeces were separated from the spent growth medium by sieving [23]; these materials were stored at $-80^{\circ}$ 

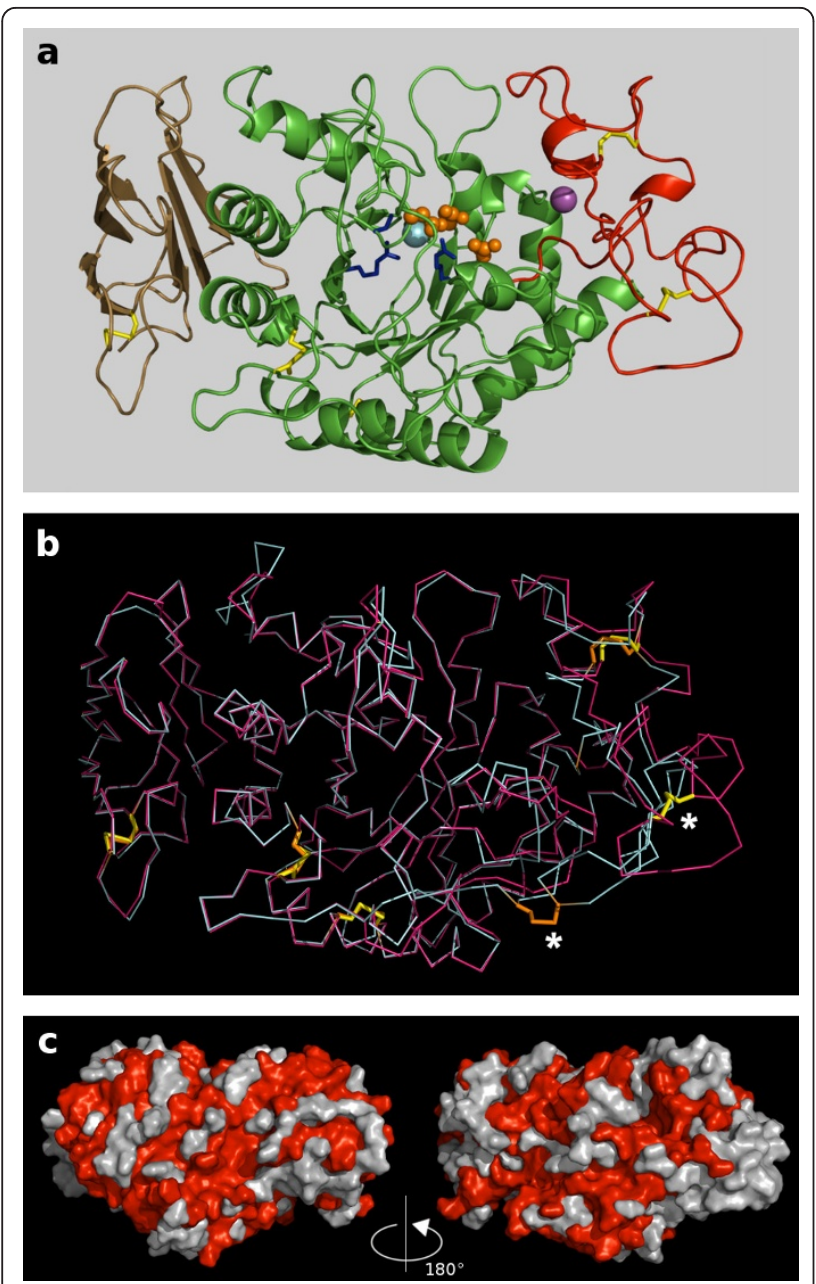

Figure 5 Spatial model of Aca $s, 4$ built by homology modeling and simulations. (a) The Aca s 4 structure (in ribbon

representation) is composed of the consensus $\alpha$-amylase domains $A$ (green), B (red), and C (brown). Three catalytic residues (D196, E232, D297) in the active site are highlighted (orange ball-and-stick). The calcium ion is depicted as a magenta sphere. The chloride binding site (blue sticks) is composed of the conserved binding residues R194 and R334 and the residue S295, which is specific for mite $\alpha$ amylases; the chloride ion is shown as a light blue sphere. The disulfide bridges are represented by yellow sticks. (b) A

superposition of C $\alpha$ traces of Aca s 4 (magenta) with HPA (cyan; PDB: 2CPU) used as a template for Aca $s 4$ modeling. The disulfide bridges are represented by yellow (Aca s 4 ) and orange (HPA) sticks; non-conserved disulfides are marked by asterisks. (c) The surface model of Aca s 4; the right-hand view is in the same orientation as in (a). The molecule is colored red for residues that are identical for Aca $s 4$ and Der $p 4$.

C. The pooled serum was prepared from serum samples collected from 33 subjects from the Czech Republic with allergies to house dust mites. The patient sera had specific IgE levels against $D$. farinae and/or D. pteronyssinus above $0.72 \mathrm{kU} / \mathrm{l}$ (scores of class 2 to 6); the titer was determined using an IgE-capture immunoenzymatic ALLERGEN System (Radim Diagnostics, Pomezia, Italy).

\section{Protein extracts}

The biological samples (mite bodies or faeces) were homogenized (50 $\mathrm{mg}$ fresh weight per $\mathrm{mL}$ ) on ice in 50 $\mathrm{mM}$ MES, pH 6.0, containing $5 \mathrm{mM} \mathrm{CaCl}_{2}, 0.1 \mathrm{M} \mathrm{NaCl}$, $25 \%$ glycerol, $0.02 \% \mathrm{NaN}_{3}$, and protease inhibitors (10 $\mu \mathrm{M}$ E-64 and $1 \mathrm{mM}$ Pefabloc). The inhibitors were not included in the extracts used for proteolytic activity measurements. The homogenate was centrifuged (10000 $\mathrm{g}, 10 \mathrm{~min}, 4^{\circ} \mathrm{C}$ ), and the supernatant was filtered with a Micropure-0.22 Separator (Millipore, Bedford, MA); the final extracts were stored at $-80^{\circ} \mathrm{C}$. The protein content was quantified by bicinchoninic acid protein assay (Pierce, Rockford, IL).

\section{Isolation of Aca s 4}

The purification procedure was based on a previously described method [21]. The whole body extract of $A$. siro was prepared in $50 \mathrm{mM} \mathrm{Na}$ acetate, $\mathrm{pH} 5.0$, containing $5 \mathrm{mM} \mathrm{CaCl}_{2}, 10 \%$ glycerol and protease inhibitors $(10 \mu \mathrm{M}$ E-64 and $1 \mathrm{mM}$ Pefabloc). Ethanol was added to a final concentration of $40 \%$, keeping the samples on ice, and the mixture was centrifuged (10000 g, $10 \mathrm{~min}, 4^{\circ} \mathrm{C}$ ). The supernatant was treated with $0.2 \%$ glycogen for $5 \mathrm{~min}$ on ice, and the $\alpha$-amylase-glycogen complex was collected by centrifugation (10000 g, 10 min, $4^{\circ} \mathrm{C}$ ) and washed with the extraction buffer containing $40 \%$ ethanol. The final sediment was incubated with rotation $\left(2 \mathrm{~h}, 26^{\circ} \mathrm{C}\right)$ in $50 \mathrm{mM} \mathrm{MES}$, pH 6.0, containing $5 \mathrm{mM} \mathrm{CaCl}_{2}$ and $10 \%$ glycerol and dialyzed against the same buffer. The purity of the isolated Aca $\mathrm{s}$ 4 was confirmed by Laemmli SDS-PAGE, and its concentration was determined by bicinchoninic acid protein assay.

\section{Proteomic methods}

Mass spectrometric characterization of Aca s 4 was performed by LC-MS/MS analysis of the tryptic digest. The LC-MS/MS analysis was performed on a LTQ Orbitrap XL hybrid mass spectrometer (Thermo Scientific, Waltham, MA) coupled to a Rheos 2000 2D capillary HPLC system (Flux instruments, Basel, Switzerland). The first dimension column was a monolithic PS-DVB $(200 \mu \mathrm{m} \times$ $10 \mathrm{~mm}$, Dionex, Sunnyvale, CA), and the second dimension column was a C18 PepMap $100(75 \mu \mathrm{m} \times 150 \mathrm{~mm}$ $\times 3 \mu \mathrm{m}$, Dionex) with gradient elution in a $0.1 \%$ formic acid/acetonitrile system. The LC-MS/MS data were processed with Sequest and Bioworks software (Thermo Scientific) and searched against the UniProt protein database http://www.uniprot.org. N-terminal Edman sequencing of Aca s 4 was performed by using a Procise 494 cLC protein sequencer (Applied Biosystems, Carlsbad, CA). The amino acid sequences were searched by BLAST http://blast.ncbi.nlm.nih.gov and aligned by ClustalW http://www.ebi.ac.uk/Tools/msa/clustalw2. 
The labeling experiment for the detection of free-thiol cysteines in Aca s 4 was performed with 5-iodoacetamidofluorescein (Molecular Probes, Eugene, OR) under denaturing conditions followed by SDS-PAGE visualization as previously described [24].

\section{Enzyme activity and inhibition assays}

$\alpha$-Amylase activity was assayed with the chromogenic substrate RBB-starch. An enzyme aliquot was incubated $\left(20 \mathrm{~min}, 26^{\circ} \mathrm{C}\right.$ ) with $0.3 \% \mathrm{RBB}$-starch in $0.1 \mathrm{M}$ BrittonRobinson buffer at the pH optimum of the enzyme (6.5 for Aca s 4 and $A$. siro extract, 7.0 for $D$. farinae extract, 6.9 for PPA) or at $\mathrm{pH} 4.5-9.0$ (pH profiling). The reaction was stopped with $0.2 \mathrm{M} \mathrm{NaOH}$, the mixture was centrifuged (10000 g, $10 \mathrm{~min}$ ), and the absorbance at $620 \mathrm{~nm}$ of the supernatant was measured against a control sample (incubated in the absence of enzyme/ extract). Typically, $0.35 \mathrm{U}$ of $\alpha$-amylase activity at the $\mathrm{pH}$ optimum was used in the assay $\left(1 \mathrm{U}\right.$ produces $\mathrm{A}_{620}$ $\mathrm{nm}=1$ ). For the activity assay in the presence of $\alpha$-amylase inhibitors, an enzyme aliquot was preincubated (20 $\min , 26^{\circ} \mathrm{C}$ ) in the assay buffer with the following inhibitor concentrations: $10 \mu \mathrm{M}$ WI-1, WI-3, or $\alpha \mathrm{AI}-1$; 0.1-10 $\mu \mathrm{M}$ acarbose; $10 \mu \mathrm{M}$ PAMI for $D$. farinae extract and $50 \mu \mathrm{M}$ PAMI for Aca s 4 and $A$. siro extract. For the activity assay in the presence of $\mathrm{NaCl}$, enzyme samples were dialyzed against water. The measurements were performed in triplicate. Proteolytic activity was assayed with the chromogenic substrate azocasein at $\mathrm{pH} 6.0$ essentially as described [25]. The inhibition of cysteine proteases in the extract was performed by preincubation (10 min, $26^{\circ} \mathrm{C}$ ) with $10 \mu \mathrm{M} \mathrm{E}-64$.

\section{Homology modeling and molecular simulations}

A 3D model of Aca s 4 was created with the SwissModel server [26] using the Aca s 4 sequence GenBank: ABL09312 and the X-ray structure of human pancreatic $\alpha$-amylase (HPA) (PDB: 2CPU) as a template. The model did not include the terminal residues 1-4 and 496 due to the lack of homology with the human enzyme (residue numbering is according to the mature Aca s 4 sequence). The $\mathrm{Ca}^{2+}$ and $\mathrm{Cl}^{-}$ions were inserted manually according to the $2 \mathrm{CPU}$ structure. Four conserved disulfide bridges (linking positions 31-87, 140-159, 376382,449-461) were constructed automatically by SwissModel, while an additional disulfide connecting Cys 113 and Cys126 was modeled using the following simulation protocol: (i) minimization of the added hydrogens, (ii) minimization and molecular dynamics of the segment 104-133: $50 \mathrm{ps}$ at $10 \mathrm{~K}$ with a restraint $(5$ to $500 \mathrm{kcal}$. $\mathrm{mol}^{-1} . \AA^{-1}$ ) on the disulfide S-S distance, (iii) molecular dynamics of the segments extending to 43-167: 50 ps at $300 \mathrm{~K}$, and (iv) minimization of the segment spanning residues 43-167. The non-conserved disulfides Cys113-
Cys126 of Aca s 4 and Cys70-Cys115 of HPA are associated with conformational differences in domain $\mathrm{B}$. The final Aca s 4 structure was validated using Molprobity [27] and deposited in the Protein Model Data Bank http://mi.caspur.it/PMDB/ under the accession code PM0077555. Structure figures were prepared with PyMOL (DeLano Scientific LLC, San Carlos, CA).

\section{Electrophoretic and immunological methods}

The proteins were separated by reducing Laemmli SDSPAGE (15\% gel), then stained with Coomassie blue or, for immunostaining transferred to a PVDF membrane by electroblotting. Immunoblots were developed using pooled patients' sera (diluted 1:1000 in $10 \mathrm{mM}$ Tris$\mathrm{HCl}, \mathrm{pH} 7.4$, containing $150 \mathrm{mM} \mathrm{NaCl}$ and $0.05 \%$ Tween 20), anti-human IgE antibody conjugated with horseradish peroxidase (Sigma) (1:25000), and SuperSignal West Pico chemiluminescent substrate (Pierce). Protein molecular mass standards (PageRuler Plus Prestained Protein Ladder, 10-250 kDa, Fermentas, Burlington, Canada) were not immunostained under these conditions. For the inhibition experiment, the pooled sera were preincubated with purified Aca s $4(1 \mu \mathrm{g} / 10 \mu \mathrm{l}$ serum). The blots were visualized with a LAS-4000 luminescent image analyzer (Fujifilm, Valhalla, NY).

\section{Abbreviations}

Aca s 4: a group 4 allergen from Acarus siro; Der $p 4$ and Der $f$ 4: a group 4 allergen from Dermatophagoides pteronyssinus and D. farinae, respectively; PPA and HPA: porcine and human pancreatic a-amylase, respectively; RBBStarch: Remazol Brilliant Blue dyed starch; PAMI: peptide a-amylase inhibitor; WI-1 and WI-3: wheat a-amylase inhibitors 1 and 3, respectively; aAl-1: bean a-amylase inhibitor 1; E-64: N-[N-(L-3-trans-carboxyirane-2-carbonyl)-L-leucyl]agmatine.

\section{Acknowledgements and Funding}

This work was supported by grant no. 525/09/P600 from the Grant Agency of the Czech Republic and by research project Z40550506. We thank Alexandr Gardavský and Jan Hubert for mite-derived materials, Eva Ohnútková for patients' sera, Zdeněk Voburka for protein sequencing, and Hillary Hoffman for critical reading of the manuscript.

\section{Authors' contributions}

JP carried out the biochemical and immunological studies. ML carried out the molecular modeling. MS performed the proteomic analysis. PT participated in the immunological analysis. LM participated in the enzyme inhibition analysis. MM designed the study and wrote the manuscript. All authors read and approved the final manuscript.

Received: 13 October 2011 Accepted: 31 January 2012 Published: 31 January 2012

\section{References}

1. Fernández-Caldas E, Iraola Calvo V: Mite allergens. Curr Allergy Asthma Rep 2005, 5:402-410.

2. Fernández-Caldas E, Iraola V, Carnés J: Molecular and biochemical properties of storage mites (except Blomia species). Protein Pept Lett 2007, 14:954-959.

3. Olsson S, van Hage-Hamsten M: Allergens from house dust and storage mites: similarities and differences, with emphasis on the storage mite Lepidoglyphus destructor. Clin Exp Allergy 2000, 30:912-919. 
4. Van der Heide $S$, Niemeijer NR, Hovenga $H$, de Monchy JG, Dubois AE, Kauffman HF: Prevalence of sensitization to the storage mites Acarus siro, Tyrophagus putrescentiae, and Lepidoglyphus destructor in allergic patients with different degrees of sensitization to the house-dust mite Dermatophagoides pteronyssinus. Allergy 1998, 53:426-430.

5. Pittner G, Vrtala S, Thomas WR, Weghofer M, Kundi M, Horak F, Kraft D, Valenta R: Component-resolved diagnosis of house-dust mite allergy with purified natural and recombinant mite allergens. Clin Exp Allergy 2004, 34:597-603.

6. Arias-Irigoyen J, Lombardero M, Arteaga C, Carpizo JA, Barber D: Limited IgE cross-reactivity between Dermatophagoides pteronyssinus and Glycyphagus domesticus in patients naturally exposed to both mite species. J Allergy Clin Immunol 2007, 120:98-104.

7. Johansson E, Johansson SGO, van Hage-Hamsten M: Allergenic characterization of Acarus siro and Tyrophagus putrescentiae and their crossreactivity with Lepidoglyphus destructor and Dermatophagoides pteronyssinus. Clin Exp Allergy 1994, 24:743-751.

8. Luczynska CM, Griffin P, Davies RJ, Topping MD: Prevalence of specific lgE to storage mites (A. siro, L. destructor and T. longior) in an urban population and cross reactivity with the house dust mite ( $D$. pteronyssinus). Clin Exp Allergy 1990, 20:403-406.

9. Puerta L, Fernandez-Caldas E, Lockey RF, Caraballo LR: Sensitization to Chortoglyphus arcuatus and Aleuroglyphus ovatus in Dermatophagoides spp. allergic individuals. Clin Exp Allergy 1993, 23:117-123.

10. Janecek S: Alpha-amylase family: molecular biology and evolution. Prog Biophys Mol Biol 1997, 67:67-97

11. MacGregor EA, Janecek S, Svensson B: Relationship of sequence and structure to specificity in the alpha-amylase family of enzymes. Biochim Biophys Acta 2001, 1546:1-20

12. Cheong N, Ramos JD, Tang CY, Chng HH, Yao R, Liang Z, Lee BW, Chua KY: Mite amylase from Blomia tropicalis (Blo t 4): differential allergenicity linked to geographical regions. Int Arch Allergy Immunol 2009, 149(1):25-32.

13. Mills KL, Hart BJ, Lynch NR, Thomas WR, Smith W: Molecular characterization of the group 4 house dust mite allergen from Dermatophagoides pteronyssinus and its amylase homologue from Euroglyphus maynei. Int Arch Allergy Immunol 1999, 120:100-107.

14. Lake FR, Ward LD, Simpson RJ, Thompson PJ, Stewart GA: Allergenicity and physicochemical characterization of house dust mite derived amylase. Int Arch Allergy Appl Immunol 1991, 94:357-358.

15. Sánchez-Monge R, García-Casado G, Barber D, Salcedo G: Interaction of allergens from house-dust mite and from cereal flours: Dermatophagoides pteronyssinus alpha-amylase (Der $\mathrm{p} \mathrm{4)}$ ) and wheat and rye alpha-amylase inhibitors. Allergy 1996, 51:176-180.

16. Hales BJ, Laing IA, Pearce $\amalg$, Hazell LA, Mills KL, Chua KY, Thornton RB, Richmond P, Musk AW, James AL, Lesouëf PN, Thomas WR: Distinctive immunoglobulin $E$ anti-house dust allergen-binding specificities in a tropical Australian Aboriginal community. Clin Exp Allergy 2007, 37:1357-1363.

17. Hubert J, Doleckova L, Hyblova J, Kudlikova I, Stejskal V, Mares M: In vitro and in vivo inhibition of a-amylases of stored-product mite Acarus siro. Exp Appl Acarol 2005, 35:281-291.

18. Numao S, Maurus R, Sidhu G, Wang Y, Overall CM, Brayer GD, Withers SG: Probing the role of the chloride ion in the mechanism of human pancreatic alpha-amylase. Biochemistry 2002, 41:215-225.

19. D'Amico S, Gerday C, Feller G: Structural similarities and evolutionary relationships in chloridedependent a-amylases. Gene 2000, 253:95-105.

20. Kluh I, Horn M, Hyblova J, Hubert J, Doleckova-Maresova L, Voburka Z Kudlikova I, Kocourek F, Mares M: Inhibitory specificity and insecticidal selectivity of a-amylase inhibitor from Phaseolus vulgaris. Phytochemistry 2005, 66:31-39.

21. Pytelkova J, Hubert J, Lepsik M, Sobotnik J, Sindelka R, Krizková I, Horn M, Mares M: Digestive alpha-amylases of the flour moth Ephestia kuehniella - adaptation to alkaline environment and plant inhibitors. FEBS J 2009, 276:3531-3546.

22. Dolecková-Maresova L, Pavlik M, Horn M, Mares M: De novo design of aamylase inhibitor: A small linear mimetic of macromolecular proteinaceous ligands. Chem Biol 2005, 12:1349-1357.

23. Erban T, Erbanova M, Nesvorna M, Hubert J: The importance of starch and sucrose digestion in nutritive biology of synanthropic acaridid mites: alpha-amylases and alpha-glucosidases are suitable targets for inhibitor- based strategies of mite control. Arch Insect Biochem Physiol 2009, 71:139-158.

24. Horn M, Baudys M, Voburka Z, Kluh I, Vondrasek J, Mares M: Free-thio Cys331 exposed during activation process is critical for native tetramer structure of cathepsin C (dipeptidyl peptidase I). Protein Sci 2002, 11:933-943.

25. Hubert J, Hyblova J, Munzbergova Z, Pekar S, Kudlikova I, DoleckovaMaresova L, Stejskal V, Mares M: Combined effect of antifeedant aamylase inhibitor and predator Cheyletus malaccensis in controlling the stored mite pest Acarus siro. Physiol Entomol 2007, 32:41-49.

26. Schwede T, Kopp J, Guex N, Peitsch MC: SWISS-MODEL: an automated protein homology-modeling server. Nucleic Acids Res 2003, 31:3381-3385.

27. Chen VB, Arendall 3rd WB, Headd JJ, Keedy DA, Immormino RM, Kapral GJ, Murray LW, Richardson JS, Richardson DC: MolProbity: all-atom structure validation for macromolecular crystallography. Acta Crystallogr D Biol Crystallogr 2010, 66(Pt 1):12-21.

doi:10.1186/1471-2091-13-3

Cite this article as: Pytelková et al:: Enzymatic activity and immunoreactivity of Aca s 4, an alpha-amylase allergen from the storage mite Acarus siro. BMC Biochemistry 2012 13:3.

\section{Submit your next manuscript to BioMed Central and take full advantage of:}

- Convenient online submission

- Thorough peer review

- No space constraints or color figure charges

- Immediate publication on acceptance

- Inclusion in PubMed, CAS, Scopus and Google Scholar

- Research which is freely available for redistribution

Submit your manuscript at www.biomedcentral.com/submit
Biomed Central 